\title{
Safety and Immunogenicity of Different Formulations of a Tetravalent Dengue Purified Inactivated Vaccine in Healthy Adults from Puerto Rico: Final Results after 3 Years of Follow-Up from a Randomized, Placebo-Controlled Phase I Study
}

\author{
Clemente Diaz, ${ }^{1}$ Michael Koren, ${ }^{2}$ Leyi Lin, ${ }^{2}$ Luis J. Martinez, ${ }^{2}$ Kenneth H. Eckels, ${ }^{2}$ Maribel Campos, ${ }^{1}$ Richard G. Jarman, ${ }^{2 \star}$ \\ Rafael De La Barrera, ${ }^{2}$ Edith Lepine, ${ }^{3}$ Irma Febo, ${ }^{1}$ David W. Vaughn, ${ }^{3}$ Todd M. Wilson, ${ }^{3}$ Robert M. Paris, ${ }^{3 *}$ Alexander C. Schmidt, ${ }^{3}$ \\ and Stephen J. Thomas ${ }^{2}$ \\ ${ }^{1}$ University of Puerto Rico School of Medicine, San Juan, Puerto Rico; ${ }^{2}$ Walter Reed Army Institute of Research, Silver Spring, Maryland; \\ ${ }^{3}$ GSK, Rockville, Maryland
}

\begin{abstract}
Four formulations of an investigational tetravalent dengue purified inactivated vaccine, administered as two doses one month (M) apart, were previously shown to be immunogenic and well-tolerated up to M13 of the phase I study NCT01702857. Here, we report results of the follow-up from M14 to year (Y) 3. One hundred healthy Puerto Rican adults, predominantly dengue virus (DENV)-primed, were randomized 1:1:1:1:1 to receive placebo or vaccine formulations: $1 \mu \mathrm{g} /$ serotype/dose adjuvanted with aluminum, AS01 $\mathrm{E}$ or $\mathrm{ASO} 3_{\mathrm{B}}$, or aluminum-adjuvanted $4 \mu \mathrm{g} / \mathrm{serotype} /$ dose. No serious adverse events occurred. Two medically-attended potential immune-mediated disease cases, vaccination unrelated, were reported (groups $1 \mu \mathrm{g}+$ Alum and $1 \mu \mathrm{g}+\mathrm{ASO} 3_{\mathrm{B}}$ ). Of 14 instances of suspected dengue, none were laboratory confirmed. Geometric mean neutralizing antibody titers against DENV 1-4 waned from M14, but remained above pre-vaccination levels for DENV 1-3, with the highest values for group $1 \mu \mathrm{g}+\mathrm{AS} 03_{\mathrm{B}}: 1220.1,920.5$, 819.4 , and 940.5 (Y2), and 1329.3, 1169.2, 1219.8, and 718.9 (Y3). All formulations appeared to be safe and immunogenic during the 3-year follow-up.
\end{abstract}

\section{INTRODUCTION}

Dengue is a vector-borne disease caused by the dengue viruses (DENVs) of the Flavivirus genus, with four serologically and genetically distinct serotypes (DENV-1, -2, -3, and -4). Although most DENV infections are mild or asymptomatic, symptomatic and severe dengue disease remains a major public health concern in tropical and subtropical regions, with a pronounced increase in incidence over the last 50 years. ${ }^{1}$ In Puerto Rico, dengue is endemic with suspected cases surpassing 10,000/year even in non-epidemic years. ${ }^{2}$

With no specific anti-DENV treatment available, prevention of dengue through immunization is considered an important approach to reduce the global burden. One live attenuated chimeric tetravalent vaccine (Dengvaxia, Sanofi Pasteur, Lyon, France) is currently licensed in 19 countries. ${ }^{1,3}$ However, the WHO recommends its administration in dengue-seropositive individuals, ${ }^{1}$ as an increased incidence of hospitalization and severe dengue was noted in dengue-seronegative vaccine recipients. ${ }^{4}$ Other investigational vaccines are under development. ${ }^{5}$

The immunogenicity and safety of an investigational tetravalent dengue purified inactivated vaccine (DPIV) formulated with different adjuvant systems have previously been reported. ${ }^{6,7}$ In a phase I clinical study conducted in a predominantly dengue-primed adult population in Puerto Rico, different formulations of $1 \mu \mathrm{g}$ per DENV type 1-4 adjuvanted with alum, $A S 01_{E}$, or $A S 03_{B}$, or $4 \mu \mathrm{g}$ per DENV type adjuvanted with alum were assessed. Three of the four formulations were shown to be highly immunogenic up to 12 months post-vaccination with two DPIV doses, and all were well tolerated. ${ }^{8}$ Here, we report final results from this trial after 3 years of follow-up.

* Address correspondence to Robert M. Paris, GSK, 14200 Shady Grove Rd., Rockville, MD 20850. E-mail: robert.m.paris@gsk.com or Richard G. Jarman, Walter Reed Army Institute of Research, 503 Robert Grant Ave., Silver Spring, MD 20910. E-mail: richard.g.jarman.mil@ mail.mil

\section{METHODS}

This phase I, randomized, observer-blind, single-center study (NCT01702857) was conducted from October 2012 to January 2018 at the University of Puerto Rico Medical Sciences Campus, Puerto Rico. The study design has been described. $^{8}$ In brief, participants were randomized $1: 1: 1: 1: 1$ to receive two doses of different formulations of DPIV, administered 4 weeks apart: $1 \mu \mathrm{g} /$ serotype/dose adjuvanted with aluminum (Group $1 \mu \mathrm{g}+$ alum), $A S 01_{E}$ (Group $1 \mu \mathrm{g}+\mathrm{AS01} 1_{\mathrm{E}}$ ) or AS03 $_{\mathrm{B}}$ (Group $1 \mu \mathrm{g}+\mathrm{ASO3}_{\mathrm{B}}$ ), $4 \mu \mathrm{g} /$ serotype/dose adjuvanted with aluminum (Group $4 \mu \mathrm{g}+$ alum), or phosphate-buffered saline as placebo. Study participants were healthy adults aged 20-39 years, living in the Caribbean for more than 10 years. The inclusion/exclusion criteria were previously detailed. ${ }^{8}$

We previously reported results through month (M) $13 .{ }^{8}$ Here, we present the final study results for year $(Y) 2$ and $Y 3$ of a 3-year follow-up, pertaining to secondary and exploratory objectives: to evaluate the safety of the four DPIV formulations from M14 to the study end, to assess exposure to DENV infection and occurrence of acute dengue illness throughout the study; and to evaluate the humoral immunogenicity of DPIV formulations to each of the four DENV types at various time points from M14 to Y3. Safety assessments were conducted for the total vaccinated cohort, comprising participants receiving at least one vaccine dose. Serious adverse events (SAEs), potential immune-mediated diseases (pIMDs), and medically attended adverse events (MAAEs) were collected by the investigator during scheduled visits or phone contacts conducted approximately every 4 months in Y2 and Y3, with serum samples collected every 4-8 months (Supplemental Figure 1).

All participants experiencing fever $\geq 38^{\circ} \mathrm{C}$ on two consecutive days were asked to contact the study staff on the second day. Following each report, the participant was invited for a visit involving a physical examination and a blood sample collection for virologic testing by reverse-transcriptase quantitative polymerase chain reaction (RT-qPCR). ${ }^{9}$ Suspected dengue was 
defined as fever (oral temperature $\geq 38^{\circ} \mathrm{C}$ ) measured at least once on two consecutive days and no alternative diagnosis with reasonable certainty. Laboratory confirmation required RTqPCR detection of DENV in blood samples. All cases of suspected and laboratory-confirmed dengue were described in detail.

Immunogenicity analyses were performed on the adapted according-to-protocol (ATP) cohort, including all evaluable participants who had data available for at least one immunogenicity end point at each time point (Supplemental Figure 1). Anti-DENV-neutralizing antibodies (NAbs) were measured using a micro-neutralization assay as previously described. ${ }^{10}$ Seropositivity was defined as a titer $\geq 10$. Seropositivity rates (defined as the percentage of seropositive participants), geometric mean titers (GMTs), and tetravalent seropositivity rates were calculated. Geometric mean titers of NAb to each DENV type were calculated by time point and group with associated asymptotic 95\% confidence intervals (Cls).

No prespecified statistical hypothesis testing was performed.

\section{RESULTS}

Of the 100 vaccinated participants, 74 completed the study. Fourteen moved/migrated from the study area, 11 were lost to follow-up, and one was enrolled in a concurrent study. The ATP cohort at M14 included 79 participants; reasons for exclusion were essential serological data missing ( $n=15)$, administration of vaccines/medication forbidden in the protocol $(n=3)$, and others $(n=3)$. Table 1 presents demographic characteristics of participants in the ATP cohort at Y3.

Safety data up to M13 have previously been published. ${ }^{8}$ From M14 to the study end, no SAEs were reported. Two pIMDs were recorded: one in the $1 \mu \mathrm{g}+$ alum group (reactive arthritis, resolved by the study end) and one in the $1 \mu \mathrm{g}+\mathrm{ASO} 3_{\mathrm{B}}$ group (rheumatoid arthritis, resolved with sequelae by the study end), with the onset at 883 and 581 days post-second vaccine dose, respectively. Both were medically attended, but were not considered as related to vaccination. No other MAAEs were reported.

Fourteen visits were conducted to evaluate suspected dengue illness for 9 participants: one in each of the $1 \mu \mathrm{g}+$ alum and $1 \mu \mathrm{g}+\mathrm{AS01} 1_{\mathrm{E}}$ groups, two in each of the $4 \mu \mathrm{g}+$ alum and $1 \mu \mathrm{g}+\mathrm{ASO}_{\mathrm{B}}$ groups, and three placebo recipients. None of the participants required hospitalization or intravenous fluids, and none of the cases evolved into an SAE. No laboratoryconfirmed dengue cases were identified. For three participants (two in the placebo group and one in the $1 \mu \mathrm{g}+\mathrm{ASO} 1_{\mathrm{E}}$ group), $a \geq 3$-fold increase in NAb titers from pre- to post-visit for evaluation of suspected dengue was observed for at least one DENV type (Table 2). One of these participants had symptoms consistent with Zika virus illness during the Zika epidemic in Puerto Rico, but testing yielded negative results for Zika, dengue, and chikungunya viruses. Subsequent development of additional clinical signs and symptoms was indicative of an alternative diagnosis for these cases (Supplemental Table 1).

NAb GMTs against DENV types 1-4 at Y2 and Y3 postvaccination waned slightly from M14 levels, but remained higher than pre-vaccination levels across investigational groups, except for DENV-4. The highest GMTs were observed in the $1 \mu \mathrm{g}+\mathrm{ASO} 3_{\mathrm{B}}$ group: 1220.1, 920.5, 819.4, and 940.5 at Y2; 1329.3, 1169.2, 1219.8, and 718.9 at Y3 for DENV types $1-4$, respectively. Increased GMTs were observed at Y3 postvaccination for placebo recipients (1222.6 [DENV-1], 780.2 [DENV-2], 1115.5 [DENV-3], and 871.6 [DENV-4]) (Figure 1). NAb seropositivity rates decreased from M14 to $Y 2$, increased slightly at the M29-31 time point, and then declined at Y3. Seropositivity rates at $\mathrm{Y} 3$ ranged from $92.3 \%$ to $100 \%$ for any of the four DENV types, in all groups except the $1 \mu \mathrm{g}+$ alum group $(78.6-85.7 \%)$. The percentages of tetravalent responders at Y3 were $78.6 \%(95 \% \mathrm{Cl}$ : 49.2-95.3) in the 1 $\mu \mathrm{g}+$ alum group, $92.9 \%$ (95\% Cl: 66.1-99.8) in the $4 \mu \mathrm{g}+$ alum group, $92.3 \%$ (95\% Cl: $64.0-99.8)$ in the $1 \mu \mathrm{g}+\mathrm{ASO1} 1_{\mathrm{E}}$ group, $100 \%$ (95\% Cl: $73.5-100)$ in the $1 \mu \mathrm{g}+\mathrm{AS03}$, and 93.8 (95\% Cl: 69.8-99.8) in participants receiving placebo. Fold changes in NAb titers for each DENV type are presented in Supplemental Figure 2.

\section{DISCUSSION}

The final results of this phase I trial of the DPIV investigational vaccine in a highly primed, dengue-endemic population extend previous findings indicating that all four evaluated formulations were well tolerated and immunogenic, with no safety concerns identified over 3 years from vaccination. Of note, this trial was not designed to explore efficacy or evaluate any differences between dengue-primed and unprimed individuals following DPIV administration.

The safety results are consistent with previous observations for the tetravalent DPIV in a first-time-in-human trial in healthy, dengue-naive adults aged 18-39 years, with all formulations being well tolerated and no SAEs observed up to 12 months post-vaccination with a two-dose series. ${ }^{7}$ Similarly, a monovalent aluminum-adjuvanted vaccine containing the same DENV-1 antigen as DPIV showed an acceptable safety profile following administration of different antigen amounts according to a two-dose schedule in healthy adults. ${ }^{6}$ In this small phase 1 study, no laboratory-confirmed dengue cases were identified during the three years of follow-up. Notably, the majority $(90 \%)$ of the participants had evidence of prior

TABLE 1

Characteristics of participants (adapted according-to-protocol cohort for immunogenicity at M14)

\begin{tabular}{|c|c|c|c|c|c|c|}
\hline & $\begin{array}{c}1 \mu \mathrm{g}+\text { alum } \\
(N=16)\end{array}$ & $\begin{array}{c}4 \mu g+\text { alum } \\
(N=16)\end{array}$ & $\begin{array}{c}1 \mu g+A S 01_{E} \\
(N=15)\end{array}$ & $\begin{array}{c}1 \mu g+\mathrm{ASO}_{\mathrm{B}} \\
(N=16)\end{array}$ & $\begin{array}{l}\text { Placebo } \\
(N=16)\end{array}$ & $\begin{array}{c}\text { Total } \\
(N=79)\end{array}$ \\
\hline Age (years) at first vaccination, mean $\pm S D$ & $26.9 \pm 4.9$ & $28.6 \pm 5.7$ & $30.1 \pm 6.7$ & $28.9 \pm 6.2$ & $28.9 \pm 6.2$ & $28.7 \pm 5.9$ \\
\hline Female, $n(\%)$ & $11(68.8)$ & $12(75.0)$ & $8(53.3)$ & $6(37.5)$ & 13 (81.3) & $50(63.3)$ \\
\hline American Hispanic/Latino ethnicity, $n$ (\%) & $16(100)$ & $16(100)$ & $15(100)$ & $16(100)$ & $16(100)$ & $79(100)$ \\
\hline $\begin{array}{l}\text { Tetravalent-positive participants at pre- } \\
\text { vaccination, } n(\%)\end{array}$ & $12(75.0)$ & $14(87.5)$ & $12(80.0)$ & $15(93.8)$ & 13 (81.3) & $66(83.5)$ \\
\hline $\begin{array}{l}\text { Tetravalent-positive participants at } \\
\text { M17-19, } n(\%)\end{array}$ & $14(87.5)$ & $15(93.8)$ & $14(93.3)$ & $16(100)$ & $13(81.3)$ & $72(91.1)$ \\
\hline
\end{tabular}


TABLE 2

Individual neutralizing antibody titers before and after suspected dengue illness for participants with suspected dengue

\begin{tabular}{|c|c|c|c|c|c|c|}
\hline No. & Group & Blood sampling* & DENV-1 & DENV-2 & DENV-3 & $\overline{\text { DENV-4 }}$ \\
\hline \multirow[t]{2}{*}{1} & Placebo & M17-M19 & 30 & 5 & 5 & 198 \\
\hline & & Y & 17 & 5 & 5 & 278 \\
\hline \multirow[t]{4}{*}{2} & Placebo & D28 & 198 & 151 & 75 & 639 \\
\hline & & D5 & 110 & 232 & 143 & 2,247 \\
\hline & & M17-M19 & 126 & 35 & 38 & 581 \\
\hline & & Y2 & 68 & 36 & 79 & 623 \\
\hline \multirow[t]{2}{*}{3} & $4 \mu g$ +alum & M7 & 38 & 81 & 35 & 56 \\
\hline & & M10 & 42 & 65 & 5 & 34 \\
\hline \multirow[t]{2}{*}{4} & $4 \mu g$ +alum & M29-31 & 5,425 & 1,632 & 1,502 & 1,199 \\
\hline & & $Y^{\prime}$ & 7 & 6 & 7 & 723 \\
\hline \multirow[t]{2}{*}{5} & $1 \mu \mathrm{g}+\mathrm{AS} 03_{\mathrm{B}}$ & M17-19 & 3,646 & 2,324 & 1,317 & 2,634 \\
\hline & & Y2 & 5,762 & 2050 & 2074 & 3,764 \\
\hline \multirow[t]{6}{*}{6} & $1 \mu \mathrm{g}+\mathrm{AS} 01_{\mathrm{E}}$ & DO & 48 & 12 & 645 & \\
\hline & & D7 & 233 & 110 & 3,345 & 28 \\
\hline & & M & 332 & 91 & 1903 & 16 \\
\hline & & M & 649 & 277 & 6,630 & 57 \\
\hline & & M1 & 342 & 131 & 2063 & 13 \\
\hline & & & 189 & 66 & 1,2 & 33 \\
\hline \multirow[t]{2}{*}{7} & $1 \mu g$ +alum & M17-19 & 600 & 547 & 460 & 66 \\
\hline & & Y2 & 380 & 579 & 530 & 55 \\
\hline \multirow[t]{2}{*}{8} & $1 \mu \mathrm{g}+\mathrm{ASO}_{\mathrm{B}}$ & M13 & 2,430 & 704 & 713 & 1,288 \\
\hline & & M17- & 1,523 & 184 & 319 & 10 \\
\hline \multirow[t]{4}{*}{9} & Placebo & M13 & 4,944 & 1,286 & 746 & 693 \\
\hline & & M17-M19 & 2,359 & 620 & 629 & 19 \\
\hline & & Y2 & 1730 & 686 & 916 & 28 \\
\hline & & M29-31 & 5,032 & 1,110 & 1,013 & 19 \\
\hline
\end{tabular}

$\mathrm{D}$ = day; D28 = 1 month post-dose 1; D56 = 1 month post-dose 2; D0 = pre-vaccination $\mathrm{D} 7=7$ days post-dose $1 ; \mathrm{DENV}=$ dengue virus; $\mathrm{M}=$ month; $\mathrm{M} 17-19=16-18$ months postdose 2; M7 = 6 months post-dose 2; M10 = 9 months post-dose $2 ; \mathrm{M} 29-31=28-30$ months post-dose $2 ; Y 3=36-38$ months post-dose 2; $M 4=3$ months post-dose 2;M13 $=12$ month post-dose $2 ; Y=36-3$ participants with suspected dengue and $a \geq 3$-fold rise in titers for at least one DENV serotype. Three participants had more than one suspect dengue visit.

${ }^{*}$ Closest time points before and after the suspected dengue visit.

DENV infection at vaccination, which limits the generalization of these results to other populations.

Consistent with previous immunogenicity data at $\mathrm{M} 13,{ }^{8}$ the $1 \mu \mathrm{g}+$ alum appeared less immunogenic than the other three formulations, with the $1 \mu \mathrm{g}+\mathrm{ASO} 3_{\mathrm{B}}$ showing the highest persistence of $\mathrm{NAb}$ at $\mathrm{Y} 3$ following vaccination. At all follow-up time points, observed GMTs against DENV types 1-4 were higher for the $1 \mu \mathrm{g}+\mathrm{ASO}_{\mathrm{B}}$ formulation, and all participants receiving $1 \mu \mathrm{g}+\mathrm{ASO}_{\mathrm{B}}$ had a tetravalent response and a positive fold change at $Y 3$. However, these data are difficult to interpret in the absence of a correlate of protection against dengue. Post-vaccination NAbs, measured by the $50 \%$ plaque reduction neutralization test, elicited by the licensed tetravalent vaccine were previously associated with vaccine efficacy against dengue. ${ }^{11}$ Although this comparison is limited by the difference in assays used, GMTs across all DENV types observed in our study indicate that increased individual antibody levels persist up to $\mathrm{Y} 3$.

An increase in NAb GMTs and seropositivity rates was observed during the last year of the follow-up, in all groups, including placebo recipients. Asymptomatic exposure to DENV or crossreactive Flavivirus during this period may be an explanation. Of note, during the study period, DENV-1 and DENV-4 were the main circulating serotypes in Puerto Rico. ${ }^{12}$ Although none of the study participants was clinically diagnosed with a Zika virus infection, Y3 coincided with a Zika virus epidemic in Puerto Rico in $2016 .^{13}$ Cross-reactivity between antibodies to Zika virus and DENV has previously been reported ${ }^{14,15}$ and is likely due to substantial similarity in the sequence type of surface glycoprotein $\mathrm{E}^{16}$
Based on these results, the $1 \mu \mathrm{g}+\mathrm{ASO}_{\mathrm{B}}$ formulation administered according to different dose schedules was assessed in a subsequent clinical trial (NCT02421367; L. Lin et al., unpublished data). Subsequently, the development of DPIV as a homologous prime-boost vaccine candidate was not pursued any further, but a heterologous prime-boost schedule using DPIV in combination with a live attenuated dengue vaccine is currently under evaluation.

\section{CONCLUSION}

Vaccination with DPIV appeared safe and immunogenic over 3 years after vaccination in an adult, dengue-endemic population.

Received June 17, 2019. Accepted for publication December 20, 2019.

Published online March 2, 2020.

Note: Supplemental figures and table appear at www.ajtmh.org.

Acknowledgments: We would like to thank the study participants and their families and all personnel at the study site for their contribution. We would also like to thank Russel Olson, Robert Putnak, Alix Collard, Vasile Coman, Elodie Tournay, Debbie Daehnick, Bruce Innis, and Francis Dessy for their contribution to the study. We thank the Modis platform for editorial assistance and manuscript coordination, on behalf of GSK. Petronela M. Petrar (Modis c/o GSK) provided medical writing assistance, and Mickaël Gaillard (Modis c/o GSK) and Janne Tys (B\&D Life Sciences c/o GSK) coordinated manuscript development and editorial support.

Financial support: This study (NCT01702857) was co-funded by the U.S. Army Medical Research and Materiel Command, Military Infectious Diseases Research Program, and GlaxoSmithKline Biologicals SA. GlaxoSmithKline Biologicals SA was involved in all stages of the study conduct and analysis and covered all costs associated with the development and publishing of this manuscript.

Disclosures: The institution of C .D., M. C., and I. F. received funding from the GSK group of companies and the U.S. Army Medical Materiel Development Activity to conduct this study. M. C. received funding from the GSK group of companies and from Merck to conduct other clinical studies. C. D. and I. F. received funding from the GSK group of companies, Merck, Takeda and Sanofi to conduct other clinical studies. T. M. W. and R. M. P. are employees of the GSK group of companies; E. L., A. C. S., and D. W. V. were employed by the GSK group of companies during the conduct of this study; R. M. P., T. M. W. and A. C. S hold shares in the GSK group of companies. M. K., L. L., K. H. E., R. D. L. B., R. G. J., S. J. T., and L. J. M. received financial and other support without any personal financial benefit from the GSK group of companies as part of the CRADA between the U.S. Army and the GSK group of companies; they also received travel support through the CRADA. K. H. E. received royalties from the license on a patented Dengue inactivated vaccine. S. J. T. received personal fees from the GSK group of companies, Chuagai Pharma, the HHS NVPO/ Tunnel Government Services and Janssen for consulting services, outside of the submitted work. S. J. T. also received personal fees for participating to scientific advisory boards for Takeda, Merck, PrimeVax and Themisbio. He is the CEO and founder of Cormac Life Sciences through which he conducts his consulting on other contracts. Finally, S. J. T. reports having an issued patent for combined flavivirus vaccines.

Authors' addresses: Clemente Diaz, Maribel Campos, and Irma Febo, University of Puerto Rico School of Medicine, San Juan, Puerto Rico, E-mails: clemente.diaz@upr.edu, maribel.campos@upr.edu, and irma.febo2@upr.edu. Michael Koren, Luis J. Martinez, Kenneth $\mathrm{H}$. Eckels, Richard G. Jarman, and Rafael De La Barrera, Walter Reed Army Institute of Research, Silver Spring, MD, E-mails: michael.a.koren2.mil@ mail.mil, luis.j.martinez.mil@mail.mil, kenneth.h.eckels.ctr@mail.mil, richard.g.jarman.mil@mail.mil, and rafael.a.delabarrera.ctr@mail.mil. Leyi Lin, National Institute of Allergy and Infectious Diseases (NIAID), 


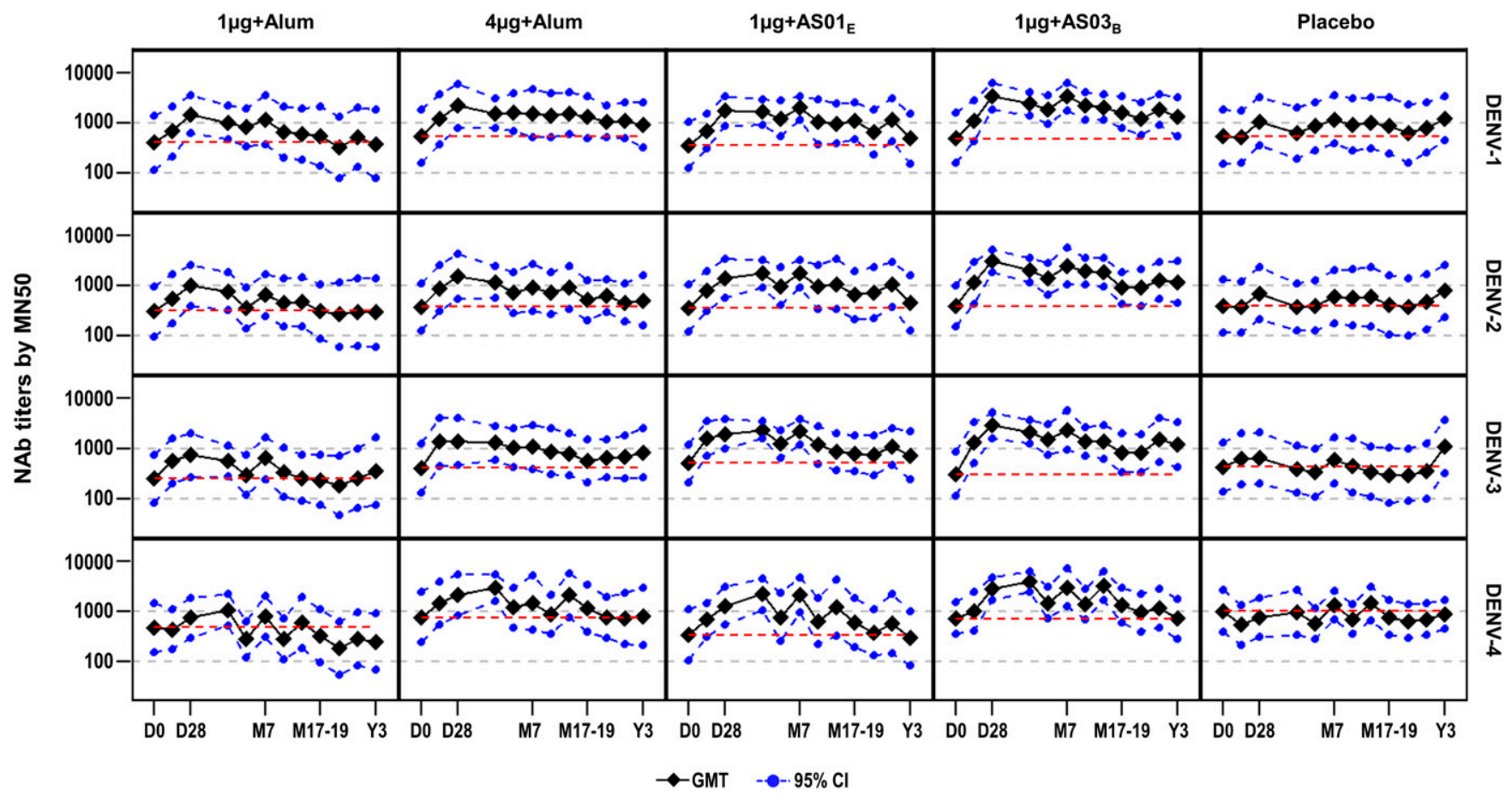

FIGURE 1. Persistence of neutralizing antibody responses up to $Y 3$ post-vaccination (adapted according-to-protocol cohort for immunogenicity). $\mathrm{D}=$ day; $\mathrm{DENV}=$ dengue virus; $\mathrm{D0}=$ pre-vaccination; $\mathrm{D} 28$ = 1 month post-dose $1 ; \mathrm{GMT}=$ geometric mean titer; $\mathrm{LL}=$ lower limit; $\mathrm{MN50}=$ microneutralization assay; $\mathrm{M}=$ month; $\mathrm{M} 7=6$ months post-dose 2; $\mathrm{M} 17-19=16-18$ months post-dose 2; $\mathrm{NAb}=$ neutralizing antibody; $\mathrm{UL}=\mathrm{upper}$ limit; $\mathrm{Y}=$ year; $\mathrm{Y} 3=36-38$ months post-dose 2 . The red dotted line represents pre-vaccination values. GMTs were computed by taking the antilog of the mean of the $\log _{10}$ titer transformations. Antibody titers below the cutoff of the assay were given an arbitrary value of half the cutoff (5).

Bethesda, MD, E-mail: leylin@gmail.com. Edith Lepine, Vetoquinol Global, Lavaltrie, Quebec, Canada, E-mail: tehdi@hotmail.com. David W. Vaughn, Bill \& Melinda Gates Foundation, Seattle, WA. E-mail: david.vaughn@gatesfoundation.org. Todd M. Wilson and Robert M. Paris, GSK, Rockville, MD, E-mail: todd.m.wilson@gsk.com and robert.m.paris@gsk.com. Alexander C. Schmidt, Bill \& Melinda Gates Medical Research Institute, Cambridge, MA, E-mail: as337y@gmail. com. Stephen J. Thomas, Division of Infectious Diseases, State University of New York, Upstate Medical University, Syracuse, NY, E-mail: thomstep@upstate.edu.

This is an open-access article distributed under the terms of the Creative Commons Attribution (CC-BY) License, which permits unrestricted use, distribution, and reproduction in any medium, provided the original author and source are credited.

\section{REFERENCES}

1. World Health Organization, 2018. Dengue vaccine: WHO position paper-September 2018. Wkly Epidemiol Rec 93: 457-476.

2. Centers for Disease Control and Prevention. Dengue in Puerto Rico. Available at http://www.cdc.gov/dengue/about/inPuerto.html. Accessed December 3, 2018.

3. Guy B, Noriega F, Ochiai RL, L'Azou M, Delore V, Skipetrova A, Verdier F, Coudeville L, Savarino S, Jackson N, 2017. A recombinant live attenuated tetravalent vaccine for the prevention of dengue. Expert Rev Vaccines 16: 1-13.

4. Sridhar S et al., 2018. Effect of dengue serostatus on dengue vaccine safety and efficacy. N Engl J Med 379: 327-340.

5. Torresi J, Ebert G, Pellegrini M, 2017. Vaccines licensed and in clinical trials for the prevention of dengue. Hum Vaccin Immunother 13: 1059-1072.

6. Martinez LJ et al., 2015. Safety and immunogenicity of a dengue virus serotype-1 purified-inactivated vaccine: results of a phase 1 clinical trial. Am J Trop Med Hyg 93: 454-460.
7. Schmidt AC et al., 2017. Phase 1 randomized study of a tetravalent dengue purified inactivated vaccine in healthy adults in the United States. Am J Trop Med Hyg 96: 1325-1337.

8. Diaz C et al., 2018. Phase I randomized study of a tetravalent dengue purified inactivated vaccine in healthy adults from Puerto Rico. Am J Trop Med Hyg 98: 1435-1443.

9. Sadon N, Delers A, Jarman RG, Klungthong C, Nisalak A, Gibbons RV, Vassilev V, 2008. A new quantitative RT-PCR method for sensitive detection of dengue virus in serum samples. $J$ Virol Methods 153: 1-6.

10. Thomas SJ et al., 2013. A phase II, randomized, safety and immunogenicity study of a re-derived, live-attenuated dengue virus vaccine in healthy adults. Am J Trop Med Hyg 88: 73-88.

11. Moodie $Z$ et al., 2018. Neutralizing antibody correlates analysis of tetravalent dengue vaccine efficacy trials in Asia and Latin America. J Infect Dis 217: 742-753.

12. Sharp TM, Ryff KR, Santiago GA, Margolis HS, Waterman SH, 2019. Lessons learned from dengue surveillance and research, Puerto Rico, 1899-2013. Emerg Infect Dis 25: 1522-1530.

13. Rosenberg R et al., 2018. Vital signs: trends in reported vectorborne disease cases-United States and Territories, 2004-2016. MMWR Morb Mortal Wkly Rep 67: 496-501.

14. Dejnirattisai $W$ et al., 2016. Dengue virus sero-cross-reactivity drives antibody-dependent enhancement of infection with Zika virus. Nat Immunol 17: 1102-1108.

15. Swanstrom JA, Plante JA, Plante KS, Young EF, McGowan E, Gallichotte EN, Widman DG, Heise MT, de Silva AM, Baric RS, 2016. Dengue virus envelope dimer epitope monoclonal antibodies isolated from dengue patients are protective against Zika virus. mBio 7: e01123-e01116.

16. Priyamvada L, Hudson W, Ahmed R, Wrammert J, 2017. Humoral cross-reactivity between Zika and dengue viruses: implications for protection and pathology. Emerg Microbes Infect 6: e33. 